\title{
Pilocytic Astrocytoma in Conus Medullaris in An Adult: A Case Report
}

\author{
Deepika Parwan ${ }^{1 *}$, Mukesh Pandey ${ }^{2}$ and Ranjan Kumar ${ }^{1}$ \\ ${ }^{1}$ Department of Pathology, Sarvodaya Hospital \& Research Centre, Faridabad, Haryana, India \\ ${ }^{2}$ Department of Neurosurgery, Sarvodaya Hospital \& Research Centre, Faridabad, Haryana, India \\ ${ }^{1}$ Department of Pathology, Sarvodaya Hospital \& Research Centre, Faridabad, Haryana, India
}

\begin{abstract}
Pilocytic astrocytoma is a rare glioma, seen predominantly in pediatric population and young adults, within first two decades of life.[1] This is often located in deep midline structures such as brainstem and cerebellum. In adults, this tumor occurs in supratentorial location [2]. It is a rare tumor and is frequently misdiagnosed and mistreated. However, being a low-grade tumor with excellent prognosis, if diagnosed correctly, it can be successfully treated by surgery only. We report a case of 45 -year-old male adult patient with conus medullaris tumor who complained of numbness and pain in the limbs and incontinence of urine and bowel. The radiology showed a sharp conus medullaris mass, which was provisionally diagnosed as low-grade glioma and was surgically resected. On intraoperative imprint cytology, it was reported as a low-grade glial tumor. Histopathological examination confirmed the diagnosis of Pilocytic astrocytoma. The patient in under follow up and doing fine.
\end{abstract}

Keywords: Pilocytic, Astrocytoma, Conus Medullaris

\section{Introduction}

Pilocytic astrocytoma was identified as a discrete entity over 70 years ago. They are predominantly benign (WHO grade1), and as a group, offer a 10-year survival rate of over $90 \% .{ }^{[3]}$ While most show classical morphology, they may present a spectrum of morphological patterns. In addition, there are difficult cases that show similarities to other gliomas, some of which are malignant and require aggressive treatment ${ }^{[4]}$.

These tumors are commonly seen in childhood, often located in deep midline structures such as the brainstem and cerebellum. ${ }^{[5]}$ Cerebellar pilocytic astrocytoma (CPA) account for approximately $30 \%$ of all pediatric central nervous system tumors and thus, constitute the second largest pediatric brain tumor group after medulloblastomas.

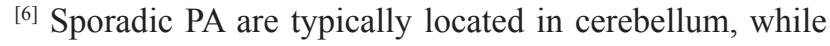
PA associated with Neurofibromatosis type1 are typically located in optic pathways. ${ }^{[7]}$

However the entire neuroaxis can be affected, with the prevalence in the hypothalamus, thalamus, optic chiasma and nerve, brainstem, cerebellum, cerebral hemispheres and basal ganglia. ${ }^{[8]} 1 / 3 \mathrm{rd}$ of the PA occur over the age of 18 years and only $17 \%$ occur at $>30$ years of age, with $50 \%$ being supratentorial in adults this being fourth documented case on review of literature.

Conus medullaris PA has been rarely reported and the disease is misdiagnosed due to its rarity. There are no clinical features that are unique to PA. Signs and symptoms usually persist for number of months, and may differ depending on size, location and presence of associated hydrocephalus. The present case being reported is of a male aged 45 years, with Conus Medullaris lesion that was accurately diagnosed on histopathology and confirmed on IHC. The patient was successfully treated by surgery.

\section{Case Report}

A 45-Year-old male was admitted to the neurosurgery dept of Sarvodaya Hospital and Research Centre, Faridabad with the chief complaints of numbness, weakness, pain in dorso-lumbar region radiating to bilateral lower limbs (Right>Left), incontinence of urine and stools.

The complaints persisted for $>2$ years and aggravated in the last 2 months. Physical examination demonstrated bilateral lower limb weakness, non-dermatomal sensory loss, bilateral lower limbs. He had been catheterized at a referring hospital.

MRI dorso-lumbar spine contrast suggested of heterogeneously enhancing lesion with indistinct margin causing fusiform expansion of lower dorsal spinal cord and Conus Medullaris. [Fig.1 and Fig.2] The lab investigations - electrolytes, protein levels, urea, creatinine, glucose, cholesterol and liver enzymes were normal. The clinical features and a mass lesion in Conus Medullaris pointed towards a primary diagnosis of low-grade glioma, that indicated that a surgical excision was required. 
Following sufficient preoperative preparation, the patient underwent a surgical treatment. The intraoperative imprint smear examination was also done which gave the diagnosis of low-grade glioma. Surgical removal of the tumor was done D12-L1 laminectomy was done, dura opened and showed evidence of multiple abnormal vessels over cord surface. Midline myelotomy was done. Lesion had good plane of separation from the cord tissue except at few places. Near total excision was done.

Tiny bit of tumor was sent for imprint cytology and on imprint cytology it was reported as a low-grade glioma. On histopathology-the tumor was reported as PA, the diagnosis was confirmed on IHC. (done outside at CORE Diagnostics) In histopathological examination - typical histology characteristics including Rosenthal fibers, loose areas, eosinophilic bodies, braid like vessels were evident [9] [ Fig.3 and Fig.4]. IHC was performed outside and markers are as follows IDH 1 -Negative, ATRX retained, p53 negative, PAS negative and ki-67 is less than $1 \%$.

\section{Discussion}

We report a case of Pilocytic astrocytoma as a mass lesion in Conus Medullaris, that too in an adult male since it is a tumor commonly seen in pediatric population.

Kumar et al ${ }^{[10]}$ also presented a case of conus medullaris pilocytic astrocytoma in 44 yrs. old female with a history of nocturnal, continuous, and burning dysesthetic low back

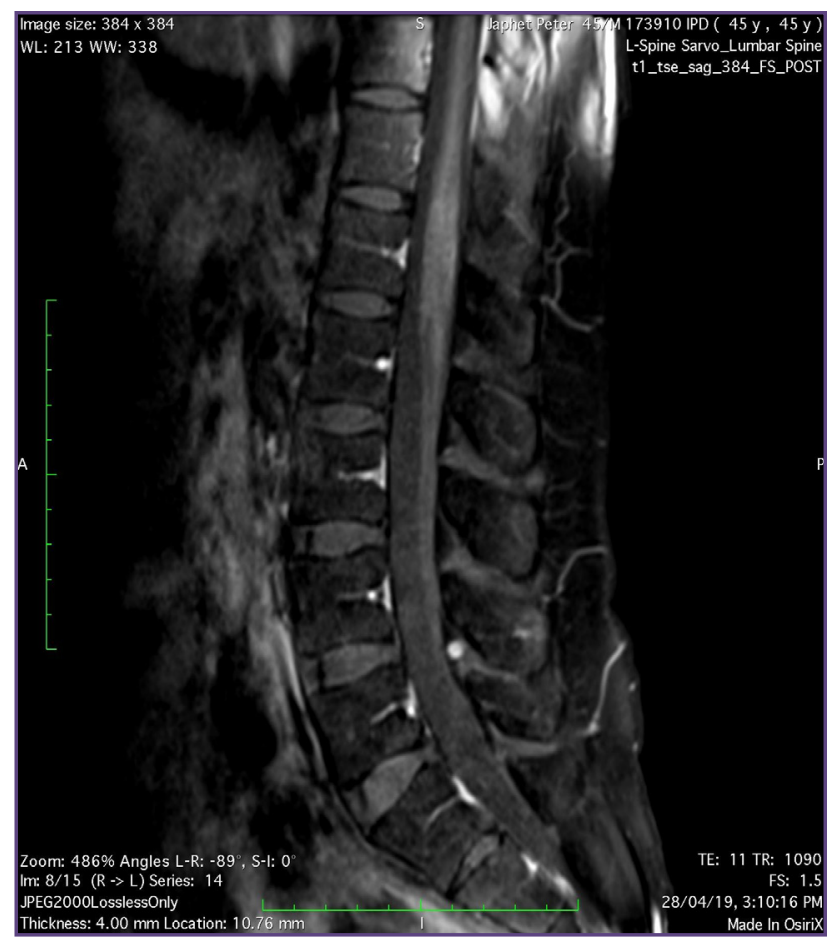

Fig. 1: T1 pre-sagittal MRI. pain radiating to both lower limbs without involvement of bladder or bowel complaints.

Lavrador JP et al ${ }^{[1]}$ also described the case of a 69 -yearold patient presenting with a broad-base gait, bilateral pain and dysesthesia of inferior limbs with a diagnosis of an intra-axial cystic lesion centered to the conus medullaris, diagnosed as pilocytic astrocytoma of conus medullaris (PACM) after surgery.

The most common tumors of Conus Medullaris are ependymoma (arising from the lining of the spinal cord) and astrocytoma (arising from the astrocytes of the spinal cord). Metastatic lesions are much less common. Involvement of the brainstem is manifested by cranial nerve palsies, decreased respiratory drive and long tract signs, which may result in tumor being unresectable. ${ }^{[12,13]}$

The pathological nature of conus medullaris tumor has a great influence on the disease prognosis. For instance, in the case of PA, complete tumor resection is curative. Therefore, careful study of preoperative MRI scans is required to reach the exact diagnosis.

\section{Conclusion}

Pilocytic astrocytoma in conus medullaris is rare tumor and best treatment is complete surgical resection, so it should be kept as differential diagnosis as complete excision is the only treatment which has very good prognosis

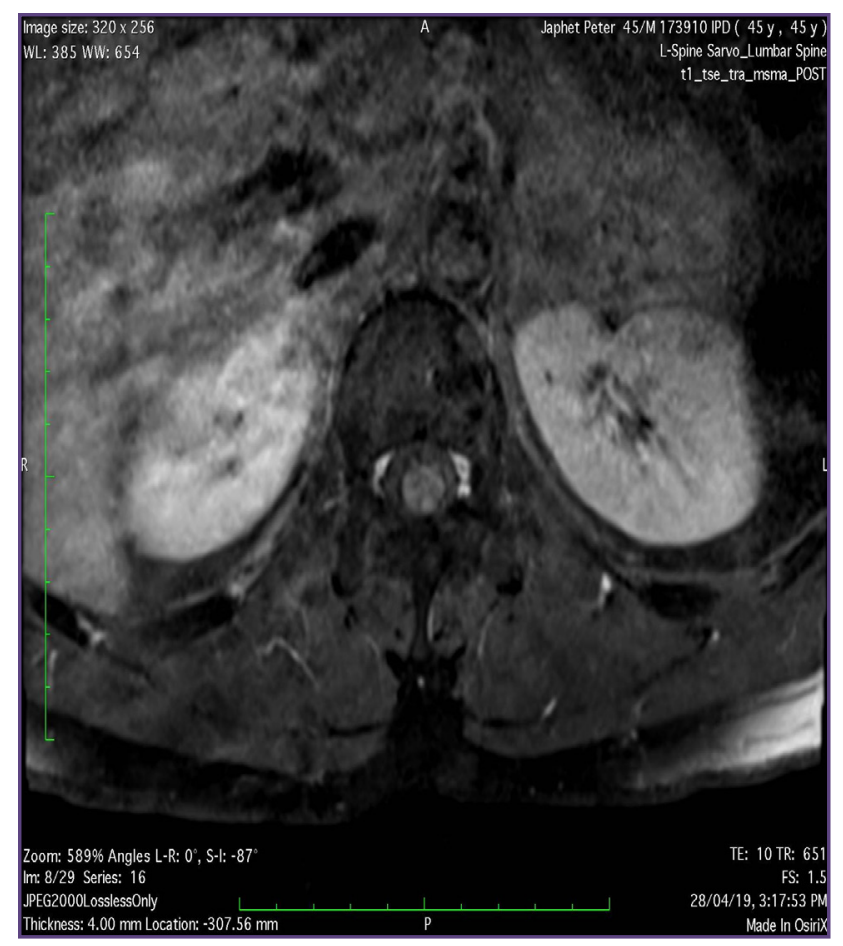

Fig. 2: T1 Post Axial MRI. 


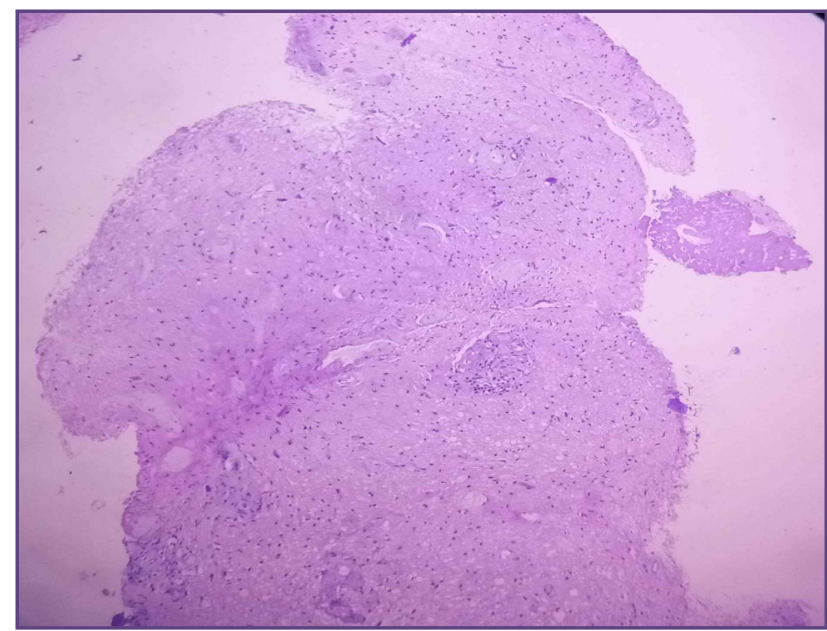

Fig. 3: Pilocytic astrocytoma 100x (H\&E stain) Arrow showing Rosenthal fibers.

\section{References}

1. Tibbetts KM, Emnett RJ, Gao F, Perry A, Gutmann DH, Leonard JR. Histopathologic predictors of pilocytic astrocytoma event-free survival. Acta Neuropathol. 2009;117:657-665.

2. Brown PD, Anderson SK, Carrero XW, O’Neill BP, Giannini C, Galanis E, Shah SA, Abrams RA, Curran WJ Jr, Buckner JC, Shaw EG. Adult patients with supratentorial pilocytic astrocytoma: long term follow-up of prospective multicenter clinical trial NCCTG-867251 (Alliance). Neurooncol Pract. 2015 Dec;2(4):199-204.

3. Cyrine S, Sonia Z, Mounir T, Badderedine S, Kalthoum T, Hedi K, Moncef M. Pilocytic astrocytoma: A retrospective study of 32 cases: Clin Neurol Neurosurg. 2012;115:1220 1225.

4. V Peter Collins, David T W Jones, Caterina Giannini, Pilocytic astrocytoma: pathology, molecular mechanisms and markers; Acta Neuropathology 2015: 129(6): 775-788.

5. Malheiros S, Diffuse low-grade astrocytomas malignancy, Neurosci Mag 1998; 2:75-80.

6. Aarsen FK, Paquier PF, Arts WF, Van Veelen ML, Michiels $\mathrm{E}$, Lequin $\mathrm{M}$ et al. Cognitive deficits and predictors 3 years after diagnosis of a pilocytic astrocytoma in childhood. J Clin Oncol. 2009;27(21):3526-32.

7. Beebe DW, Ris MD, Armstrong FD, Fontanesi J, Mulhern R, Holmes E et al. Cognitive and adaptive outcome in low-grade paediatric cerebellar astrocytomas: evidence of diminished

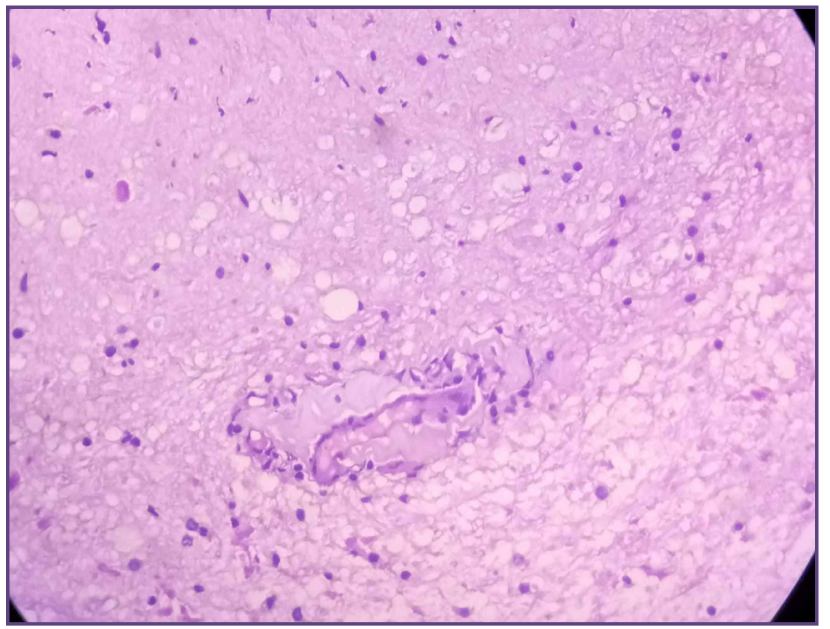

Fig. 4: Pilocytic astrocytoma 400x. (H\&E stain) showing Rosenthal fibers, loose areas, eosinophilic bodies, braid like vessels.

cognitive and adaptive functioning in National Collaborative Research Studies. J Clin Oncol. 2005;23(22):5198-204.

8. Hayostek CJ, Shaw EG, Scheithauer B, O'Fallon JR, Weiland TL, Schomberg PJ, Kelly PJ, Hu TC.Astrocytomas of the cerebellum. A Comparative Clinicopathologic Study of Pilocytic and Diffuse Astrocytomas. Cancer. 1993;72(3):856-69.

9. CBTRUS(Central Brain Tumor Registry of the United States ) statistical Report: Primary Brain and Central Nervous System Tumors diagnosed in the US in 2004-2008: Central Brain Tumor Registry of the US, 2012:

10. Kumar A, Shah RM, Gupta N. Rare tumor of conus medullaris in an adult with a favorable outcome. Journal of Surgical Technique and Case Report. 2012 Jan;4(1):67-68.

11. Lavrador JP, Oliveira E, Pimentel J, Livraghi S. Adult pilocytic astrocytoma of conus medullaris: clinical considerations and review of the literature. CNS Oncol. 2017 Apr;6(2):107-110.

12. Burkhard C, Di Patre PL, Schüler D, Schüler G, Yaşargil MG, Yonekawa Y, Lütolf UM, Kleihues P, Ohgaki H. A population-based study of the incidence and survival rates in patients with pilocytic astrocytoma. J Neurosurg. 2003;98:1170-1174.

13. Nair AP, Mehrotra A, Das KK, Srivastava AK, Sahu RN, Kumar R. Clinico-radiological profile and nuances in the management of cervicomedullary junction intramedullary tumors. Asian J Neurosurg. 2014;9:21-28.

*Corresponding author:

Dr Deepika Parwan, Sarvodaya Hospital \& Research Centre, YMCA road, Sector -8, Faridabad, Haryana, India

Phone: +919711811080

Email: deepikaparwan@gmail.com 